Arch. Tierz., Dummerstorf 50 (2007) 2, 204-213

Department of Commodity Science and Animal Improvement, University of Warmia and Mazury in Olsztyn, Poland

KATARZYNA KLECZEK, ELŻBIETA WILKIEWICZ-WAWRO, KAZIMIERZ WAWRO and WŁODZIMIERZ MAKOWSKI

\title{
Effect of body weights of day-old Muscovy ducklings on growths and carcass traits
}

\begin{abstract}
The purpose of this work was to determine the effect of the body weights of day-old Muscovy ducklings on some slaughter value traits of females aged ten weeks and males aged twelve weeks. The relationships between the body weights of day-old ducklings, daily gains and slaughter value of grown-up ducks were also analyzed. The experimental materials comprised 96 White Muscovy ducklings (46 $\sigma^{\lambda}$ and 50 P ). Males and females were reared separately, to twelve and ten weeks of age respectively. At the completion of the rearing period the birds were fasted for 12 hours and sacrificed. The carcasses were chilled, weighed and subjected to a detailed dissection. Before statistical calculations were carried out, males and females were divided into five weight groups, depending on their body weights on the first day following hatching. At the end of the experiment it was found that the grown-up ducks once classified as light, medium-heavy and heavy ducklings did not differ significantly in terms of body weight, mean daily gains, carcass weight, as well as the weight and percentage of particular tissue components in a carcass. Low and statistically non-significant values of the coefficients of simple correlation and regression suggest a lack of interdependences between the body weights of day-old ducklings and the slaughter value parameters of adult Muscovy ducks. The only exception was the carcass dressing percentage of males, which was found to be significantly negatively correlated with their body weights on the first day of life.
\end{abstract}

Key Words: Muscovy ducks, body weight, slaughter value, coefficients of regression and simple correlation

\section{Zusammenfassung}

Titel der Arbeit: Einfluss des Körpergewichtes von Eintagsküken auf Schlachtkörpermerkmale bei männlichen bzw. weiblichen Warzenenten im Alter von 10 bzw. 12 Wochen

Untersucht wurde der Einfluss des Körpergewichtes von Eintagsküken der Weißen Warzenenten (Cairina moschata) auf Wachstums- und Schlachtkörpermerkmale. Die Schlachtung von 46 männlichen Tieren erfolgte im Alter von 12, die der 50 weiblichen Tiere mit 10 Wochen. Ermittelt wurden die Beziehungen zwischen dem Eintagsgewicht und den Wachstumsbzw. Schlachtkörpermerkmalen. Die nach Geschlechtern getrennt gehaltenen Tiere wurden nach einer 12-stündigen Nüchterungszeit geschlachtet, gewogen und zerlegt. Es wurden abhängig vom Eintagsgewicht fünf Auswertungsgruppen gebildet. Die Gruppen mit dem geringsten Eintagsgewicht unterschieden sich hinsichtlich des Wachstums und der einzelnen Schlachtkörpermerkmale nicht von den Tieren der anderen Gruppen, was auf nicht vorhandene Abhängigkeiten hinweist. Eine Ausnahme bildete die negative Beziehung des Eintagsgewichtes der männlichen Tiere zum prozentualen Ausschlachtungsergebnis.

Schlüsselwörter: Warzenenten, Körpergewicht, Schlachtleistung, der Regressionskoeffizient, Korrelationskoeffizient

\section{Introduction}

The body weight of a day-old hatchling is dependent on a variety of factors, including the age of the parental flock (VIEIRA and MORAN, 1999), time of staying in the hatching unit, and - most importantly - egg weight (HALEVY et al., 2004; BADOWSKI et al., 2005; BORUTA and KOBYLIŃSKA, 2005). Birds hatched from small and medium-sized eggs are generally lighter that those hatched from heavy eggs. The body weight of a newly-hatched bird may be also considerably affected by the genotype of parents. According to WITTMANN (1997), heavy-line goslings are 
characterized by higher initial body weights and significantly higher final body weights, as compared with medium- and light-line ones.

WILSON (1991) reported that in the 1980s a $1 \mathrm{~g}$ increase in the body weights of newly-hatched broilers resulted in a $8-13 \mathrm{~g}$ increase in their final body weights. Due to the breeding progress observed in this area, these values still display a growing tendency. Thus, the best production results can be expected of the heaviest hatchlings. However, the findings of other authors indicate that the initial body weights of birds do not always decide about their slaughter value. WAWRO et al. (1988) analyzed White Italian geese and found that at the completion of raising grown-ups once classified as light and heavy goslings did not differ significantly in terms of body weight and carcass tissue composition. HALEVY et al. (2004) pointed out to various proportions of the digestive tract in the body weight of broiler chickens with different birth weights. In broilers that developed from heavier chicks the digestive tract had a lower percentage in the total body weight, as compared with those that developed from lighter chicks.

Many breeders share the opinion that light hatchlings have a low biological potential and are characterized by a slow growth rate, which results in worse production results of broilers. It follows that chicks hatched from small eggs should not be used for broiler production (BONDARI and KAZENI, 1975). On the other hand, GUILL and WASHBURN (1973) and WASHBURN and GUILL (1974) reported that the hatch weights of birds had an insignificant effect on their further growth and development. The purpose of this work was to determine the effect of the body weights of day-old Muscovy ducklings on some slaughter value traits of females aged ten weeks and males aged twelve weeks. The relationships between the body weights of day-old ducklings, daily gains and slaughter value of grown-up ducks were also analyzed.

\section{Materials and Methods}

The experimental materials comprised 96 White Muscovy ducklings (46 $\lesssim$ and 50 क ), with breeding stock imported from France ( $\widehat{\jmath}$ Cabreur $\mathrm{x} q$ Casablanca). The breeding of Muscovy ducks in Poland is generally based on ducklings imported from France. The ducks that served as the experimental materials in the present study are currently available on the Polish market. The day the ducklings were brought from the Poultry Plant to the Animal Laboratory of the Department of Commodity Science and Animal Improvement, University of Warmia and Mazury in Olsztyn, was treated as the first day of their life, since no more than $12 \mathrm{~h}$ had passed since the onset of hatching. Males were reared to twelve weeks of age, and females - to ten weeks of age. Sexed ducklings were weighed, marked and placed in two pens, males and females separately The birds were kept indoors at the Animal Laboratory, as recommended by Groupe Grimaud Fréres (GRIMAUD FRÉRES, 2003), and fed ad libitum commercial diets: a starter diet (from one day to three weeks of age) containing $22.51 \%$ crude protein (CP) and 12.31 MJ metabolizable energy (ME), and a grower diet (from four weeks of age to the end of the experimental period) containing 20.07\% CP and 12.79 MJ ME)

The ducks were weighed at two-week intervals. At the completion of the rearing period the birds were fasted for 12 hours and sacrificed by cervical dislocation. Following slaughter, the ducks were bled for about five min., and then scalded in water at about $63^{\circ} \mathrm{C}$ for $1 \mathrm{~min}$., to facilitate plucking. The birds were defeathered manually. Then the carcasses were eviscerated (removing the digestive tract, lungs, 
trachea, heart, abdominal fat and crop), and the heads and feet were cut off (the heads were cut off between the occipital condyle and the atlas, and the feet were cut off at the carpal joint). Then the carcasses were chilled at $+4^{\circ} \mathrm{C}$ for 12 hours, weighed and divided into the following parts:

1. neck - obtained by cutting along the line joining the cephalic borders of the coracoids;

2. wings - obtained by cutting through the shoulder joints;

3. legs - obtained by cutting through the hip joints (from the pubic process, through the groin towards the back, and then along the backbone, starting from the anterior border of the pelvis); to separate the thigh from the drumstick, a cut was made through the knee joint;

4. breast quarter - obtained by a double cut through the cartilaginous junctures of the ribs, from the inferior border of the backbone towards the coracoids;

5. dorsal-lumbar quarter - the remaining part of the carcass.

The carcass parts were weighed and dissected into meat, bones, skin with subcutaneous fat, and intermuscular fat. Lean weight is the weight of muscular tissue without intermuscular fat, which was separated during dissection. The weight of skin with fat includes skin and a layer of subcutaneous fat (since in poultry, especially in waterfowl, they are joined together and difficult to separate), intermuscular fat and abdominal fat. Bone weight includes the weights of all bone elements of the carcass, separated during detailed dissection. In addition, breast muscles, Pectoralis major and Pectoralis minor, were dissected from the breast quarter by cutting along the breastbone crest, clavicle and coracoids, and along the line of the attachment of these muscles to the ribs. The tissue components were weighed accurate to $0.1 \mathrm{~g}$. The percentages of particular tissue components in carcass weight were calculated as ratios between the weight of a given component and cold carcass weight.

The carcass dressing percentage was calculated according to the formula:

carcass dressing

percentage $=$

weight of a chilled carcass with neck and giblets x 100\%

body weight

Before statistical calculations were carried out, males and females were divided into five weight groups, depending on their body weights on the first day following hatching: males - group I - very light hatchlings ( $\leq 49.8$ g), group II - light hatchlings (49.9 - 52.1 g), group III - medium-heavy hatchlings (52.2 - 54.4 g), group IV heavy hatchlings (54.5 - 56.7), group V - very heavy hatchlings ( $\geq 56.8 \mathrm{~g})$; females group I - very light hatchlings ( $\leq 48.7$ g), group II - light hatchlings (48.8 - $50.8 \mathrm{~g})$, group III - medium-heavy hatchlings (50.9 - $52.9 \mathrm{~g})$, group IV - heavy hatchlings (53.0 - 55.0), group V - very heavy hatchlings ( $\geq 55.1 \mathrm{~g})$. The number of males and females in particular groups is presented in Tables 1 and 2. Due to sexual dimorphism observed in Muscovy ducks as well as a different duration of the rearing period, the calculations were done separately for males and females.

The statistical analysis included:

- statistical characteristics of the traits examined - arithmetic means $(\bar{x})$ and standard deviation (SD); 
- determination of the significance of differences between birds of particular singlesex weight groups (1-way ANOVA);

- calculation of the coefficients of simple correlation $(r)$ and regression $\left(b_{y_{\mathrm{i}} \mathrm{x}}\right)$ between the body weights of day-old ducklings (x) and some slaughter value traits of grown-up ducks $\left(\mathrm{y}_{\mathrm{i}}\right)$.

Statistical calculations were performed using STATISICA software (StatSoft, 2001).

\section{Results}

The mean body weights of day-old males are shown in Table 1 . The differences between the groups were statistically significant and observed until the end of the third week. From four weeks to the end of the rearing period no differences were recorded in body weight between males of particular weight groups. At twelve weeks of age the mean body weights of males ranged from $5034 \mathrm{~g}$ in group I to $5384 \mathrm{~g}$ in group V.

Table 1

Body weights (g) of Muscovy ducks at different weeks of the rearing period (Körpergewicht (g) männlicher und weiblicher Warzenenten in den einzelnen Aufzuchtwochen)

\begin{tabular}{|c|c|c|c|c|c|c|c|}
\hline \multirow[b]{2}{*}{ Specification } & \multirow[b]{2}{*}{ Sex } & \multirow[b]{2}{*}{ 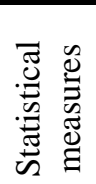 } & \multicolumn{5}{|c|}{ Weight groups } \\
\hline & & & $\begin{array}{c}\mathrm{I} \\
\leq 49.8 \mathrm{~g}\end{array}$ & $\begin{array}{c}\text { II } \\
49.9-52.1 \mathrm{~g}\end{array}$ & $\begin{array}{c}\text { III } \\
52.2-54.4 \mathrm{~g}\end{array}$ & $\begin{array}{c}\text { IV } \\
54.5-56.7 \mathrm{~g}\end{array}$ & $\begin{array}{c}\mathrm{V} \\
\geq 56.8 \mathrm{~g}\end{array}$ \\
\hline \multirow[t]{2}{*}{ Number of birds } & $\widehat{\sigma}$ & & 10 & 11 & 9 & 8 & 8 \\
\hline & 우 & & 14 & 7 & 13 & 7 & 9 \\
\hline \multicolumn{8}{|l|}{$\begin{array}{l}\text { Weeks of the } \\
\text { rearing period }\end{array}$} \\
\hline \multirow{4}{*}{ Day 1} & & $\overline{\mathrm{X}}$ & $47.6^{\mathrm{A}}$ & $50.9^{\mathrm{B}}$ & $53.3^{\mathrm{C}}$ & $55.4^{\mathrm{D}}$ & $59.5^{\mathrm{E}}$ \\
\hline & 0 & SD & 1.5 & 0.8 & 0.5 & 0.8 & 2.2 \\
\hline & 0 & $\overline{\mathrm{X}}$ & $47.5^{\mathrm{A}}$ & $49.8^{\mathrm{B}}$ & $51.9^{\mathrm{C}}$ & $54.4^{\mathrm{D}}$ & $58.3^{\mathrm{E}}$ \\
\hline & + & SD & 1.3 & 0.7 & 0.8 & 0.7 & 2.6 \\
\hline \multirow{4}{*}{ Week: 2} & $\lambda$ & $\overline{\mathrm{X}}$ & $480.0^{\mathrm{a}}$ & $509.1^{\mathrm{ab}}$ & $483.3^{\mathrm{a}}$ & $521.3^{\mathrm{b}}$ & $506.3^{\mathrm{ab}}$ \\
\hline & 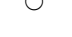 & SD & 27.5 & 25.9 & 29.2 & 34.8 & 38.5 \\
\hline & 0 & $\overline{\mathrm{X}}$ & 416.0 & 410.0 & 434.6 & 430.0 & 426.7 \\
\hline & 7 & SD & 25.6 & 22.3 & 33.0 & 32.2 & 28.0 \\
\hline \multirow{4}{*}{4} & $\lambda$ & $\overline{\mathrm{X}}$ & 1611.0 & 1698.2 & 1620.0 & 1722.5 & 1666.3 \\
\hline & 0 & SD & 91.9 & 113.1 & 80.5 & 123.7 & 86.3 \\
\hline & 0 & $\overline{\mathrm{X}}$ & 1209.6 & 1192.8 & 1256.5 & 1232.9 & 1244.0 \\
\hline & 7 & SD & 78.8 & 74.7 & 68.1 & 82.1 & 76.4 \\
\hline \multirow{4}{*}{6} & $\lambda$ & $\overline{\mathrm{X}}$ & 2950.0 & 3144.4 & 2974.4 & 3112.4 & 3065.7 \\
\hline & 0 & SD & 243.7 & 226.3 & 205.3 & 230.8 & 137.9 \\
\hline & 0 & $\overline{\mathrm{X}}$ & 1940.0 & 1913.3 & 1972.0 & 1965.0 & 1999.0 \\
\hline & 7 & SD & 153.5 & 132.2 & 139.2 & 162.1 & 149.5 \\
\hline \multirow{2}{*}{8} & $\lambda$ & $\bar{X}$ & 3957.6 & 4250.9 & 4048.9 & 4153.8 & 4141.3 \\
\hline & 0 & SD & 341.7 & 373.8 & 311.3 & 331.7 & 184.7 \\
\hline \multirow{6}{*}{10} & 0 & $\overline{\mathrm{X}}$ & 2505.4 & 2492.2 & 2562.5 & 2525.7 & 2570.5 \\
\hline & 7 & SD & 187.9 & 155.0 & 165.5 & 171.6 & 137.8 \\
\hline & $\lambda$ & $\overline{\mathrm{X}}$ & 4720.0 & 5073.6 & 4830.0 & 4866.3 & 4933.8 \\
\hline & 0 & SD & 287.3 & 450.0 & 372.9 & 404.3 & 285.8 \\
\hline & 0 & $\bar{X}$ & 2772.0 & 2782.9 & 2816.9 & 2756.7 & 2874.4 \\
\hline & 7 & SD & 247.3 & 181.0 & 194.3 & 221.0 & 154.2 \\
\hline \multirow{2}{*}{12} & $\lambda$ & $\overline{\mathrm{X}}$ & 5034.0 & 5223.6 & 5228.9 & 5215.0 & 5383.8 \\
\hline & 0 & SD & 373.1 & 503.1 & 381.3 & 435.5 & 320.2 \\
\hline
\end{tabular}


Table 2

Daily gains (g) of Muscovy ducks at different weeks of the rearing period (Tägliche Zunahmen (g) männlicher und weiblicher Warzenenten in den einzelnen Aufzuchtwochen)

\begin{tabular}{|c|c|c|c|c|c|c|c|}
\hline \multirow[b]{2}{*}{$\begin{array}{l}\text { Weeks of the } \\
\text { rearing period }\end{array}$} & \multirow[b]{2}{*}{ Sex } & \multirow{2}{*}{ 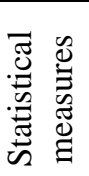 } & \multicolumn{5}{|c|}{ Weight groups } \\
\hline & & & $\begin{array}{c}\mathrm{I} \\
\leq 49.8 \mathrm{~g}\end{array}$ & $\begin{array}{c}\text { II } \\
49.9-52.1 \mathrm{~g}\end{array}$ & $\begin{array}{c}\text { III } \\
52.2-54.4 \mathrm{~g}\end{array}$ & $\begin{array}{c}\text { IV } \\
54.5-56.7 \mathrm{~g}\end{array}$ & $\begin{array}{c}\mathrm{V} \\
\geq 56.8 \mathrm{~g}\end{array}$ \\
\hline \multirow{4}{*}{$0-2$} & $\lambda$ & $\bar{X}$ & 30.9 & 32.7 & 30.7 & 33.3 & 31.9 \\
\hline & 0 & SD & 1.9 & 1.8 & 2.1 & 2.5 & 2.7 \\
\hline & م & $\bar{X}$ & 26.2 & 25.7 & 27.3 & 26.8 & 26.3 \\
\hline & 7 & SD & 1.6 & 1.6 & 2.4 & 2.3 & 2.0 \\
\hline \multirow{4}{*}{$3-4$} & $\lambda$ & $\overline{\mathrm{X}}$ & 80.8 & 84.9 & 81.2 & 85.8 & 82.3 \\
\hline & $\delta$ & SD & 5.5 & 6.8 & 4.0 & 7.0 & 4.2 \\
\hline & 0 & $\overline{\mathrm{X}}$ & 57.5 & 57.3 & 58.2 & 56.9 & 56.9 \\
\hline & + & SD & 4.3 & 2.4 & 4.0 & 3.9 & 4.0 \\
\hline \multirow{4}{*}{$5-6$} & $\lambda$ & $\overline{\mathrm{X}}$ & 95.6 & 103.3 & 96.7 & 99.3 & 99.9 \\
\hline & 0 & SD & 11.3 & 9.5 & 10.0 & 8.0 & 4.8 \\
\hline & 0 & $\overline{\mathrm{X}}$ & 52.4 & 53.1 & 49.9 & 52.1 & 54.4 \\
\hline & 7 & SD & 7.7 & 5.6 & 7.6 & 6.2 & 8.0 \\
\hline \multirow{4}{*}{$7-8$} & $\lambda$ & $\overline{\mathrm{X}}$ & 71.9 & 79.0 & 76.7 & 74.4 & 76.9 \\
\hline & ó & SD & 13.9 & 11.7 & 8.5 & 8.5 & 9.1 \\
\hline & 0 & $\overline{\mathrm{X}}$ & 39.8 & 39.7 & 43.0 & 37.3 & 42.6 \\
\hline & 7 & SD & 6.8 & 6.8 & 5.9 & 5.0 & 6.2 \\
\hline \multirow{4}{*}{$9-10$} & $\lambda$ & $\overline{\mathrm{X}}$ & 54.5 & 58.8 & 55.8 & 50.9 & 56.6 \\
\hline & $\delta$ & SD & 13.1 & 14.5 & 7.5 & 12.6 & 9.6 \\
\hline & 0 & $\overline{\mathrm{X}}$ & 18.6 & 19.4 & 19.0 & 19.9 & 21.0 \\
\hline & 7 & SD & 5.3 & 4.4 & 4.4 & 7.1 & 3.4 \\
\hline 1112 & $\lambda$ & $\overline{\mathrm{X}}$ & 22.4 & 32.1 & 28.5 & 24.9 & 32.1 \\
\hline $11-12$ & 0 & SD & 10.0 & 12.2 & 8.5 & 6.9 & 5.4 \\
\hline
\end{tabular}

Table 3

Results of the slaughter analysis of Muscovy males (Ergebnisse der Schlachtkörperzerlegung männlicher Enten)

\begin{tabular}{|c|c|c|c|c|c|c|}
\hline \multirow[b]{2}{*}{ Specification } & \multirow{2}{*}{ 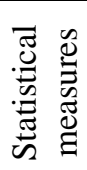 } & \multicolumn{5}{|c|}{ Weight groups } \\
\hline & & $\begin{array}{c}\mathrm{I} \\
\leq 49.8 \mathrm{~g}\end{array}$ & $\begin{array}{c}\text { II } \\
49.9-52.1 \mathrm{~g}\end{array}$ & $\begin{array}{c}\text { III } \\
52.2-54.4 \mathrm{~g}\end{array}$ & $\begin{array}{c}\text { IV } \\
54.5-56.7 \mathrm{~g}\end{array}$ & $\begin{array}{c}\mathrm{V} \\
\geq 56.8 \mathrm{~g}\end{array}$ \\
\hline \multicolumn{7}{|l|}{ Weight (g) of: } \\
\hline \multirow[t]{2}{*}{ Carcass } & $\bar{X}$ & 3734.1 & 3930.9 & 3732.8 & 3784.5 & 3785.3 \\
\hline & SD & 264.4 & 348.4 & 300.3 & 329.1 & 332.6 \\
\hline \multirow{2}{*}{ Breast muscles } & $\overline{\mathrm{X}}$ & 728.8 & 776.5 & 739.3 & 758.1 & 779.8 \\
\hline & SD & 98.4 & 81.5 & 40.3 & 69.7 & 95.0 \\
\hline \multirow[t]{2}{*}{ Total meat } & $\overline{\mathrm{X}}$ & 1893.5 & 2007.2 & 915.2 & 1954.5 & 1987.5 \\
\hline & SD & 167.9 & 118.1 & 107.2 & 138.6 & 190.9 \\
\hline \multirow[t]{2}{*}{ Fat with skin } & $\overline{\mathrm{X}}$ & 1004.4 & 1059.5 & 996.0 & 976.8 & 954.8 \\
\hline & SD & 132.3 & 195.3 & 174.6 & 160.5 & 98.9 \\
\hline \multirow{2}{*}{ Bones } & $\overline{\mathrm{X}}$ & 496.9 & 509.0 & 481.9 & 518.5 & 507.3 \\
\hline & SD & 34.7 & 32.3 & 32.9 & 36.4 & 57.8 \\
\hline \multirow{3}{*}{$\begin{array}{l}\text { Percentage in a carcass of: } \\
\text { Breast muscles }\end{array}$} & & & & & & \\
\hline & $\bar{X}$ & 19.52 & 19.79 & 19.84 & 20.07 & 20.56 \\
\hline & SD & 2.26 & 1.69 & 0.65 & 1.52 & 1.11 \\
\hline \multirow[t]{2}{*}{ Total meat } & $\overline{\mathrm{X}}$ & 50.71 & 51.24 & 51.41 & 51.76 & 52.48 \\
\hline & SD & 2.65 & 2.96 & 2.02 & 2.43 & 0.95 \\
\hline \multirow[t]{2}{*}{ Fat with skin } & $\bar{X}$ & 26.85 & 26.79 & 26.54 & 25.70 & 25.21 \\
\hline & SD & 2.55 & 2.92 & 2.73 & 2.50 & 0.86 \\
\hline \multirow[t]{2}{*}{ Bones } & $\overline{\mathrm{X}}$ & 13.34 & 12.99 & 12.95 & 13.75 & 13.40 \\
\hline & SD & 0.93 & 0.70 & 0.93 & 0.96 & 0.99 \\
\hline \multirow{2}{*}{$\begin{array}{l}\text { Carcass dressing percentage } \\
\text { (\%) }\end{array}$} & $\overline{\mathrm{X}}$ & 76.54 & 76.22 & 76.10 & 76.07 & 74.65 \\
\hline & SD & 2.36 & 1.72 & 1.21 & 1.83 & 4.59 \\
\hline
\end{tabular}


Between two and ten weeks no differences were recorded in body weight between females of particular weight groups.

Table 2 present the mean daily gains of males and females respectively in successive weeks of the experimental period. Both in the group of males and females the daily gains of birds with low, medium and high initial body weights were comparable.

The body weights of day-old ducklings had no significant effect of the slaughter value traits of adults (Tables 3 and 4). However, the highest weight of a carcass (3930.9 g), meat (2007.2 g) as well as fat with skin (1059.5 g) was recorded in 12-week-old males once classified as light ducklings (group II, Table 3). The relative content of lean, breast muscles, fat and bones in the carcasses of males representing different weight groups was at a similar level. The carcass dressing percentage, expressed as a ratio between the weight of a chilled carcass with giblets and live body weight, ranged between $74.65 \%$ in the group of very heavy males (V) to $76.54 \%$ in the group of very light males (I). In females (Table 4) the highest weights of a carcass and tissue components at the end of rearing (ten weeks) were recorded in group $\mathrm{V}$ (very heavy ducklings), but this difference was statistically non-significant. Both the proportions of particular tissue components in a carcass and the carcass dressing percentage were comparable in all groups of females (Table 4).

Table 4

Results of the slaughter analysis of Muscovy females (Ergebnisse der Schlachtkörperzerlegung weiblicher Tiere)

\begin{tabular}{|c|c|c|c|c|c|c|}
\hline \multirow[b]{2}{*}{ Specification } & \multirow{2}{*}{ 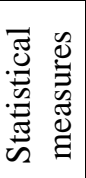 } & \multicolumn{5}{|c|}{ Weight groups } \\
\hline & & $\begin{array}{c}\mathrm{I} \\
\leq 49.8 \mathrm{~g}\end{array}$ & $\begin{array}{c}\text { II } \\
49.9-52.1 \mathrm{~g}\end{array}$ & $\begin{array}{c}\text { III } \\
52.2-54.4 \mathrm{~g}\end{array}$ & $\begin{array}{c}\text { IV } \\
54.5-56.7 \mathrm{~g}\end{array}$ & $\begin{array}{c}\mathrm{V} \\
\geq 56.8 \mathrm{~g}\end{array}$ \\
\hline Weight (g) of: & & & & & & \\
\hline Carcass & $\begin{array}{l}\bar{X} \\
\text { SD }\end{array}$ & $\begin{array}{c}1970.0 \\
173.7\end{array}$ & $\begin{array}{c}1977.7 \\
123.4\end{array}$ & $\begin{array}{c}2008.7 \\
131.4\end{array}$ & $\begin{array}{c}1991.4 \\
166.1\end{array}$ & $\begin{array}{c}2042.3 \\
108.4\end{array}$ \\
\hline Breast muscles & $\begin{array}{l}\bar{X} \\
\text { SD }\end{array}$ & $\begin{array}{c}372.7 \\
43.8\end{array}$ & $\begin{array}{c}372.1 \\
26.1\end{array}$ & $\begin{array}{c}379.3 \\
24.8\end{array}$ & $\begin{array}{c}374.7 \\
29.0\end{array}$ & $\begin{array}{c}390.6 \\
27.9\end{array}$ \\
\hline Total meat & $\begin{array}{c}\bar{X} \\
\text { SD }\end{array}$ & $\begin{array}{c}962.5 \\
88.1\end{array}$ & $\begin{array}{c}962.2 \\
45.6\end{array}$ & $\begin{array}{c}980.9 \\
66.7\end{array}$ & $\begin{array}{c}979.1 \\
55.8\end{array}$ & $\begin{array}{c}998.0 \\
49.0\end{array}$ \\
\hline Fat with skin & $\begin{array}{l}\bar{X} \\
\text { SD }\end{array}$ & $\begin{array}{c}563.7 \\
75.4\end{array}$ & $\begin{array}{c}580.0 \\
79.0\end{array}$ & $\begin{array}{c}577.2 \\
80.0\end{array}$ & $\begin{array}{l}586,1 \\
107.2\end{array}$ & $\begin{array}{c}597,2 \\
77.8\end{array}$ \\
\hline Bones & $\begin{array}{l}\bar{X} \\
\text { SD }\end{array}$ & $\begin{array}{c}248.2 \\
16.5\end{array}$ & $\begin{array}{c}246.6 \\
9.4\end{array}$ & $\begin{array}{c}251.7 \\
19.0\end{array}$ & $\begin{array}{c}239.7 \\
12.1\end{array}$ & $\begin{array}{c}254.9 \\
7.8\end{array}$ \\
\hline Percentage in a carcass of: & & & & & & \\
\hline Breast muscles & $\begin{array}{l}\bar{X} \\
\text { SD }\end{array}$ & $\begin{array}{c}18.91 \\
1.38\end{array}$ & $\begin{array}{c}18.83 \\
1.05\end{array}$ & $\begin{array}{c}18.91 \\
1.07\end{array}$ & $\begin{array}{c}18.87 \\
1.53\end{array}$ & $\begin{array}{c}19.14 \\
1.19\end{array}$ \\
\hline Total meat & $\begin{array}{l}\bar{X} \\
\text { SD }\end{array}$ & $\begin{array}{c}48.89 \\
2.17\end{array}$ & $\begin{array}{c}48.71 \\
1.48\end{array}$ & $\begin{array}{c}48.88 \\
2.47\end{array}$ & $\begin{array}{c}49.29 \\
2.48\end{array}$ & $\begin{array}{c}48.93 \\
2.37\end{array}$ \\
\hline Fat with skin & $\begin{array}{c}\bar{X} \\
\text { SD }\end{array}$ & $\begin{array}{c}28.55 \\
2.10\end{array}$ & $\begin{array}{c}29.23 \\
2.44\end{array}$ & $\begin{array}{c}28.68 \\
2.84\end{array}$ & $\begin{array}{c}29.28 \\
3.45\end{array}$ & $\begin{array}{c}29.15 \\
2.62\end{array}$ \\
\hline Bones & $\begin{array}{l}\bar{X} \\
\text { SD }\end{array}$ & $\begin{array}{c}12.65 \\
0.82\end{array}$ & $\begin{array}{c}12.50 \\
0.76\end{array}$ & $\begin{array}{c}12.53 \\
0.63\end{array}$ & $\begin{array}{c}12.08 \\
0.82\end{array}$ & $\begin{array}{c}12.50 \\
0.55\end{array}$ \\
\hline $\begin{array}{l}\text { Carcass dressing percentage } \\
(\%)\end{array}$ & $\begin{array}{l}\bar{X} \\
\text { SD }\end{array}$ & $\begin{array}{c}75.85 \\
1.23\end{array}$ & $\begin{array}{c}75.35 \\
1.87\end{array}$ & $\begin{array}{c}75.56 \\
0.86\end{array}$ & $\begin{array}{c}75.51 \\
1.38\end{array}$ & $\begin{array}{c}75.69 \\
1.14\end{array}$ \\
\hline
\end{tabular}

A significant correlation between the body weights of Muscovy males and the initial weights of ducklings was noted at two weeks of age only $\left(r_{x y}=0.356\right.$; Table 5). In subsequent weeks the coefficients of simple correlation between these traits were low and non-significant, which indicates the lack of interdependence between them. This 
was also confirmed by low values of regression coefficients $\left(\mathrm{b}_{\mathrm{yx}}\right)$. A $1 \mathrm{~g}$ increase in the body weight of newly-hatched ducklings permitted to increase the body weight of males aged twelve weeks by $19.5 \mathrm{~g}$ only (Table 5). An analysis of the coefficients of simple correlation and regression between the mean daily gains of males and the body weights of day-old ducklings revealed that the body weights of day-old ducklings had no significant effect on this trait, either (Table 5).

Table 5

Coefficients of regression and simple correlation between body weights on the first day (x) and body weights and daily gains of Muscovy ducks $\left(\mathrm{y}_{\mathrm{i}}\right)$ in successive weeks of the rearing period (Regressions- und Korrelationskoeffizienten von Eintagsgewicht $(\mathrm{x})$ und täglichen Zunahmen ( $\mathrm{y}_{\mathrm{i}}$ ) in darauf folgenden Lebenswochen männlicher und weiblicher Tiere)

\begin{tabular}{|c|c|c|c|c|}
\hline \multirow{3}{*}{ Dependent variables $\left(\mathrm{y}_{\mathrm{i}}\right)$} & \multirow{2}{*}{\multicolumn{2}{|c|}{$\frac{\delta^{\lambda}}{\text { coefficients of }}$}} & \multirow{2}{*}{\multicolumn{2}{|c|}{$\begin{array}{c}q \\
\text { coefficients of }\end{array}$}} \\
\hline & & & & \\
\hline & regression $b_{\mathrm{yx}}$ & correlation $\mathrm{r}_{\mathrm{xy}}$ & regression $b_{y x}$ & correlation $r_{x y}$ \\
\hline \multicolumn{5}{|l|}{ Weight (g): } \\
\hline Week 2 & $2.8^{*}$ & $0.356 *$ & 1.1 & 0.165 \\
\hline 4 & 5.9 & 0.238 & 0.7 & 0.049 \\
\hline 6 & 10.1 & 0.194 & 0.9 & 0.027 \\
\hline 8 & 14.6 & 0.191 & 4.4 & 0.112 \\
\hline 10 & 14.5 & 0.164 & 8.2 & 0.166 \\
\hline 12 & 19.5 & 0.191 & - & - \\
\hline \multicolumn{5}{|l|}{ Daily gains (g) during (weeks): } \\
\hline Day 1 - week 2 & 0.1 & 0.239 & 0.0 & 0.014 \\
\hline $3-4$ & 0.2 & 0.163 & 0.0 & -0.032 \\
\hline $5-6$ & 0.3 & 0.135 & 0.0 & 0.009 \\
\hline $7-8$ & 0.3 & 0.129 & 0.3 & 0.161 \\
\hline $9-10$ & 0.0 & -0.010 & 0.3 & 0.230 \\
\hline $11-12$ & 0.4 & 0.155 & - & - \\
\hline
\end{tabular}

* - significance of the coefficients of regression (b) or correlation (r) at $\mathrm{P} \leq 0.05$

Similarly as in males, also in females no significant correlation was found between the body weights of newborns and the values of this trait in subsequent weeks (Table 5). The values of simple correlation coefficients were low ( $\mathrm{r}$ from 0.027 to 0.166). The correlation between the mean daily gains of females over the experimental period and the body weights of day-old ducklings was non-significant (Table 5). Greater initial weights of ducklings did not result in greater daily gains for the first five weeks. 
Between six and ten weeks a theoretical increase in the body weight of a day-old duckling by $1 \mathrm{~g}$ could potentially increase daily gains by $0.3 \mathrm{~g}$ only.

Table 6

Coefficients of regression and simple correlation between body weights on the first day (x) and slaughter value traits of Muscovy ducks $\left(\mathrm{y}_{\mathrm{i}}\right)$ (Regressions- und Korrelationskoeffizienten von Eintagsgewicht (x) und Schlachtkörpermerkmalen $\left(\mathrm{y}_{\mathrm{i}}\right)$ männlicher und weiblicher Tiere)

\begin{tabular}{|c|c|c|c|c|}
\hline \multirow{3}{*}{ Dependent variables $\left(\mathrm{y}_{\mathrm{i}}\right)$} & \multirow{2}{*}{\multicolumn{2}{|c|}{\begin{tabular}{c}
\multicolumn{1}{c}{} \\
coefficients of
\end{tabular}}} & \multirow{2}{*}{\multicolumn{2}{|c|}{$\begin{array}{c}+ \\
\text { coefficients of } \\
\end{array}$}} \\
\hline & & & & \\
\hline & regression $b_{\mathrm{yx}}$ & correlation $r_{x y}$ & regression $b_{y x}$ & correlation $r_{x y}$ \\
\hline \multicolumn{5}{|l|}{ Weight (g) of: } \\
\hline Carcass & -9.7 & -0.147 & 5.7 & 0.156 \\
\hline Breast muscles & 0.8 & 0.046 & 1.3 & 0.152 \\
\hline Total meat & -0.4 & -0.012 & 3.1 & 0.175 \\
\hline Fat with skin & -8.0 & -0.245 & 2.7 & 0.133 \\
\hline Bones & -0.8 & -0.099 & 0.5 & 0.117 \\
\hline \multicolumn{5}{|l|}{ Percentage in a carcass of: } \\
\hline Breast muscles & 0.07 & 0.211 & 0.01 & -0.036 \\
\hline Total meat & 0.12 & 0.240 & 0.01 & -0.026 \\
\hline Fat with skin & -0.14 & -0.277 & 0.05 & 0.076 \\
\hline Bones & 0.01 & 0.053 & -0.06 & -0.085 \\
\hline Carcass dressing percentage & $-0.16^{*}$ & $-0.295 *$ & 0.00 & 0.004 \\
\hline
\end{tabular}

* - significance of the coefficients of regression (b) or correlation (r) at $\mathrm{P} \leq 0.05$

The coefficients of simple correlation and regression contained in Table 6 indicate that the slaughter value parameters of males aged twelve weeks and females aged ten weeks were not related to the body weights of day-old ducklings. The values of the regression coefficients from Table 6 show that a theoretical increase in the body weight of a newly-hatched Muscovy male by $1 \mathrm{~g}$ may cause a decrease in the weight of a carcass, meat, fat and bones by $9.7 \mathrm{~g}, 0.4 \mathrm{~g}, 8 \mathrm{~g}$ and $0.8 \mathrm{~g}$ respectively, as well as an increase in breast muscle weight by $0.8 \mathrm{~g}$. The percentages of the above tissue components in a carcass are also dependent on the initial body weights of males to a slight degree only (Table 6). A significant correlation was observed between the independent variable and the carcass dressing percentage $\left(r_{x y}=-0.295\right)$. It follows that an increase in the body weight of a newborn male by $1 \mathrm{~g}$ will cause a decrease in the carcass dressing percentage by $0.16 \%$, so at the completion of the rearing period the carcass dressing percentage of males that developed from heavier (by e.g. 10 g) ducklings may be as much as $1.6 \%$ lower as compared with the carcass dressing percentage of males that developed from lighter ones. 
The body weights of day-old females had no considerable impact on such traits as carcass weight, the weights and percentages of meat, breast muscles, fat with skin and bones (Table 6). The coefficients of simple correlation between the independent variable and the parameters analyzed ranged between -0.085 to 0.175 , i.e. were low and non-significant. The values of regression coefficients were comparable, which suggests that the use of heavier ducklings is not followed by a marked improvement in their slaughter value.

\section{Discussion}

The results of the study show that the final body weights of birds classified into the groups of light, medium-heavy and heavy ducklings were comparable (Table 1). The mean body weights of males aged twelve weeks and females aged ten weeks were similar to or higher than those reported by other authors (LEWCZUK et al., 1992; GÓRSKI and WITAK, 1995; BAEZA et al., 1998).

The daily gains of Muscovy ducks representing different weight groups were also comparable throughout the rearing period (Table 2). Somewhat different results were achieved in an experiment on White Italian geese (WAWRO et al., 1988), where the body weight gains of light and heavy goslings were similar, while medium-heavy ones were characterized by the lowest daily gains.

The body weights of day-old ducklings had no effect on carcass weight and on the weights and percentages of tissue components (Tables 3 and 4). The carcass dressing percentage of both males and females was comparable in all groups, and varied from 74.65 to $76.54 \%$. A similar carcass dressing percentage of Muscovy ducks (approx. 74\%) was observed by WAWRO et al. (2004), and a slightly lower (approx. 73\%) by LEWCZUK et al. (1992). WAWRO et al. (1988) demonstrated that in geese both carcass weight and the weights of particular tissue components were the highest in birds classified as heavy, while the relative proportions of lean, fat and bones in a carcass were comparable in all weight groups. HALEVY et al. (2004) found that breast muscle weight was significantly higher in broiler chickens hatched from heavy eggs than in those hatched from light eggs. On the other hand, egg weight as well as hatchling weight had no influence on carcass fatness.

The coefficients of regression $\left(\mathrm{b}_{\mathrm{yx}}\right)$ and simple correlation (r) between the body weights of day-old ducklings and the slaughter value traits of Muscovy ducks, given in Tables 5 and 6, were found to be low and non-significant in the majority of cases. This suggests that not only medium-heavy and heavy, but also very light and light hatchlings are suitable for production. In our previous studies high initial body weights of Muscovy ducks had no significant effect on an increase in their final body weights and the content of particular tissue components in a carcass. This is generally consistent with the results obtained for geese (WAWRO et al., 1988).

It may be concluded that the grown-up ducks once classified as light, medium-heavy and heavy ducklings did not differ significantly in terms of body weight, mean daily gains, carcass weight, as well as the weight and percentage of particular tissue components in a carcass at the end of the experiment it. Low and statistically nonsignificant values of the coefficients of simple correlation and regression suggest a lack of interdependences between the body weights of day-old ducklings and the slaughter value traits of adult Muscovy ducks. The only exception was the carcass dressing percentage of males, which was found to be significantly negatively 
correlated with their body weights on the first day of life. This indicates that even very light Muscovy ducklings ( $\hat{\sigma}<50 \mathrm{~g}, \uparrow<49 \mathrm{~g}$ ) can be used as broilers.

\section{References}

BAÉZA, E.; SALICHON, M.R.; MARCHÉ, G.; JUIN, H.:

Effect of sex on growth, technological and organoleptic characteristics of the Muscowy duck breast muscle. Brit. Poultry Sci., 39 (1998), 398-403

BADOWSKI, J.; BIELIŃSKA, H.; PAKULSKA, E.; WOLC, A.:

Relationships between some traits of hatching eggs and the body weight of growing geese. Proc. XVII ${ }^{\text {th }}$ International Poultry Science Symp. (PB WPSA), Poznań, Poland, (2005), 13-14

BONDARI, K.; KAZENI, R.:

The influence of egg size on initial chick weight and subsequent broiler growth. Arch. Geflügelk., 12 (1975), 3-52

BORUTA, A.; KOBYLIŃSKA, J.:

Effect of hen egg weight on the results of incubation and weight of the hatched chicks. Proc. XVII ${ }^{\text {th }}$ International Poultry Science Symp. (PB WPSA), Poznań, Poland, (2005), 21-22

GÓRSKI, J.; WITAK, B.:

Comparative investigations of the growth of body weight, fleshing and fatness in Muscovy ducks and crossbreds (Muscovy x Pekin) (in Polish). Zesz. Nauk. WSRP Siedl., Drob., 40 (1995), 159-169

GRIMAUD FRÉRES: http//www.grimaud.com. Accessed April (2003).

GUILL, R.A.; WASHBURN, K.W.:

Relationship between hatch weight as percentage of egg weight and feed conversion ratio in broiler chick. Poult. Sci., 52 (1973), 1641-1646

HALEVEY, O.; HEIFETZ, S; SKLAN, D.:

Heavier chicks at hatch have improved marketing body weight by enhancing skeletal muscle growth but not intestinal development. Proc. XXII ${ }^{\text {th }}$ World's Poultry Congress, Stambul, Turkey, (2004), CD.

LEWCZUK, A.; BOCHNO, R.; WAWRO, E.:

Slaughter value of Muscovy and Pekin of A-44 strain ducks (in Polish). Pr. Mater. Zoot., 42 (1992), 8390

STATISTICA:

StatSoft, Inc., version 6 (2001), www.statsoft.com.

VIEIRA, S.L.; MORAN JR, E.T.:

Effects of egg origin and chick post-hatch nutrition on broiler life performance and meat yields. World's Poult. Sci. J. 55 (1999), 127-142

WASHBURN, K.W.; GUILL, R.A.:

Relationship of embryo weight as percentage of egg weight to efficiency of feed utilization in the hatchen chick. Poult. Sci., 54 (1974); 760-769

WAWRO, K.; BOCHNO, R.; WAWRO, E.; RAJKOWSKA, H.:

Influence of body weight of one day old goslings on some slaughter values of twelve week old White Italian geese (in Polish). Acta Acad. Agricult. Techn. Olst., Zoot. 31 (1988), 171-179

WAWRO, K.; WILKIEWICZ-WAWRO, E.; KLECZEK, K.; BRZOZOWSKI, W.:

Slaughter value and meat quality of Muscovy and Pekin ducks and their crossbreds and evaluation of heterosis effects. Arch. Tierz., Dummerstorf 47 (2004), 287-299

WILSON, H.R.:

Interrelationships of egg size, hitch size, posthatching growth and hatchability. World's Poult. Sci. J. 47 (1991), 5-16

WITTMANN, M.:

Influence of age, sex and genotype on fattening performance, slaughtering results, and meat quality of geese on intensive feeding. Proc. $11^{\text {th }}$ European Symp. on Waterfowl, Nantes, France, (1997), 561-568

Received: 2006-05-09

Accepted: 2006-12-11

Author's address

Dr. KATARZYNA KLECZEK*, Prof. ELŻBIETA WILKIEWICZ-WAWRO,

Prof. KAZIMIERZ WAWRO, Mgr. WŁODZIMIERZ MAKOWSKI

Department of Commodity Science and Animal Improvement,

University of Warmia and Mazury, ul. Oczapowskiego 5,

OLSZTYN, POLAND

*Corresponding Author

E-Mail: katarzyna.kleczek@uwm.edu.pl 